\title{
Alexandre Gordon, ВЕЛИКАЯ ФРАНЦУЗСКАЯ РЕВОЛЮЦИЯ В СОВЕТСКОЙ ИСТОРИОГРАФИИ [La Grande Révolution française dans l'historiographie soviétique]
}

\section{Varoujean Poghosyan}

\section{(2) OpenEdition \\ Journals}

Édition électronique

URL : https://journals.openedition.org/ahrf/11913

DOI : 10.4000/ahrf.11913

ISSN : 1952-403X

Éditeur :

Armand Colin, Société des études robespierristes

\section{Édition imprimée}

Date de publication : 1 décembre 2010

Pagination : 193-194

ISBN : 978-2-200-92634-2

ISSN : 0003-4436

Référence électronique

Varoujean Poghosyan, « Alexandre Gordon, Великая французская революция в советской историографии [La Grande Révolution française dans l'historiographie soviétique] », Annales historiques de la Révolution française [En ligne], 362 I octobre-décembre 2010, mis en ligne le 25 mars 2011, consulté le 23 avril 2022. URL : http://journals.openedition.org/ahrf/11913 ; DOI : https:// doi.org/10.4000/ahrf.11913

Ce document a été généré automatiquement le 23 avril 2022

Tous droits réservés 


\title{
Alexandre Gordon, ВЕЛИКАЯ ФРАНЦУЗСКАЯ РЕВОЛЮЦИЯ В СОВЕТСКОЙ ИСТОРИОГРАФИИ [La Grande Révolution française dans l'historiographie soviétique]
}

\author{
Varoujean Poghosyan
}

\section{RÉFÉRENCE}

Alexandre Gordon, ВЕЛИКАЯ ФРАНЦУЗСКАЯ РЕВОЛЮЦИЯ В СОВЕТСКОЙ ИСТОРИОГРАФИИ [La Grande Révolution française dans l'historiographie soviétique], Moscou, Naouka, 2009, 384 p.

1 De grands historiens russes et soviétiques, tels N. Karéiev, N. Loukine, V. Daline, V. Dounaïevski et d'autres ont minutieusement étudié la contribution de leurs prédécesseurs aux études révolutionnaires. Le récent livre d'Alexandre Gordon, élève d'Y. Zakher, diffère cependant de cette littérature, car il nous en présente une nouvelle vision. Comme l'auteur le note dans sa préface, il ne s'agit pas d'une approche traditionnelle, mais d'une approche culturelle et historique, autrement dit, théorique, qui permet de redécouvrir bien des particularités de l'évolution de la pensée historique en URSS, ainsi que de discuter de manière détaillée les mutations assez compliquées qui ont bouleversé la science historique soviétique.

2 On sait que la politisation de la science historique en URSS était beaucoup plus forte qu'ailleurs. Alexandre Gordon a ainsi le mérite de discuter l'œuvre de ses prédécesseurs en prenant pour cadre les changements idéologiques ayant conditionné l'évolution des positions des historiens, en fonction des interprétations des fondateurs de la théorie marxiste et des instructions des chefs du Parti Communiste (les unes et les autres étant 
devenues pour eux des «idées fixes »). C'est l'idée principale du livre. En se référant à l'évolution de la politique, l'auteur distingue ainsi trois étapes de l'historiographie révolutionnaire soviétique: les années 1920, les années 1930-1950 et les années 1950-1980 (p. 15-16).

Dans un premier temps, Alexandre Gordon analyse la position des bolcheviks. Après l'instauration de leur pouvoir, ils ont conçu la Révolution française comme un événement "prototype» de celui de 1917 et ont utilisé son expérience (surtout l'exemple de la Terreur jacobine), notamment afin de justifier leurs répressions lors de la guerre civile. Selon l'auteur, c'est la conception historiographique typique de la première décennie de l'existence du pouvoir soviétique (p. 25). Il a sans doute raison d'affirmer que cette historiographie s'est formée dans les années 1920 exceptionnellement dans le cadre de la méthodologie marxiste. Il souligne par ailleurs l'un des traits spécifiques de la nouvelle science: pour la majorité des historiens de cette époque (N. Loukine, V. Daline et d'autres), souvent acteurs de la révolution de 1917, la Révolution française était devenue un moyen de mobilisation des forces politiques pour la défense de la révolution russe (p. 29).

En dépit de l'attitude univoque des historiens à l'égard de la Terreur, que je viens de mentionner, Alexandre Gordon conclut qu'ils s'en tenaient grosso modo à l'interprétation classique de la Révolution, en soulignant son caractère bourgeois. Il note que parmi les trois aspects propres à la science soviétique (le matérialisme économique, le déterminisme des classes et la foi en l'omnipotence du pouvoir), celui de la signification progressiste de la lutte des classes occupait une position prépondérante : «L'idéologie a piloté la méthodologie », conclut-il (p.49). Il n'est pas étonnant que, d'après les jugements avancés en 1926 par N. Boukharine, ayant opposé la Révolution française à la révolution de 1917 (dans le cas de la première il avait souligné le désaccord entre les objectifs capitalistes de la Révolution et la petite bourgeoisie, son moteur; il s'agissait de la crainte d'une perspective d'un « Thermidor soviétique »), les historiens de l'URSS aient renoncé à recourir à des analogies historiques, comme ils le faisaient auparavant, entre ces deux révolutions, en traitant la Révolution française de "prototype » de la révolution russe. Cette circonstance les a obligés à réviser leur position à l'égard de la Révolution française en la qualifiant de révolution « antipode».

5 L'historiographie soviétique de cette époque a identifié le caractère bourgeois de la Révolution à sa dimension antiféodale. Dans ces conditions, il était logique que les années 1930 voient se développer les études sur l'activité politique des Jacobins, comme représentants les plus déterminés de la classe révolutionnaire de leur époque. Certes, cette particularité de la science soviétique avait eu une autre raison, que l'auteur explique : les chercheurs tâchaient de motiver, à l'exemple des Jacobins, la nécessité de la dictature du Parti Communiste en URSS. Or, postérieurement, on étudia essentiellement l'activité des Jacobins en relevant leur rôle dans le processus d'écrasement de la féodalité et du pouvoir absolu (d'après la terminologie de cette époque) et du remplacement d'une des formes de l'exploitation (féodale) par une autre (capitaliste). Alexandre Gordon note à juste raison que cette circonstance a entravé certaines études, notamment dans le domaine de la contre-révolution. L'auteur de ces lignes peut constater par ailleurs les difficultés qu'il avait eu à surmonter lors de la publication de ses articles sur l'histoire de la contre-révolution à l'époque du Directoire. 
6 Alexandre Gordon insiste sur certains traits de la science soviétique : l'absence de pluralisme, la domination de la méthodologie marxiste (dans sa version soviétique bien sûr dont le fondateur a été Staline), la réalisation des études à partir du seul point de vue de la lutte des classes. Avec les années 1920 et 1930, l'auteur montre que ces caractères se renforcent ; les historiens soviétiques devaient suivre la ligne générale du Parti Communiste élaborée par Staline. On les obligeait de mener une lutte acharnée contre toutes les théories "bourgeoises ». Pour l'auteur, il s'agit là d'un nouveau type de science historique, qu'il qualifie d'« arme idéologique » (p. 86-88). À partir du milieu des années 1930, cependant, les vues de Staline concernant l'histoire changent ; il lance alors l'idée de la confrontation de la révolution socialiste avec la révolution bourgeoise, en définissant finalement la Révolution française non seulement comme un "antipode» de la révolution russe, mais en précisant également ses limites chronologiques de 1789 à 1794. De là dérive l'absence presque complète d'intérêt en URSS envers l'histoire post-thermidorienne et directoriale; de là, aussi, souligne Alexandre Gordon, procède le destin cruel de nombreux historiens soviétiques: G. Fridland a été fusillé, N. Loukine est mort en prison, Y. Zakher, V. Daline et bien d'autres ont été exilés comme « ennemis du peuple ».

7 Après la mort de Staline, malgré quelques changements idéologiques, les freins essentiels au libre développement de la pensée historique sont maintenus (il s'agit surtout de la monopolisation des recherches historiques par la théorie marxiste). Quant à l'idéologie du Parti Communiste, nul ne pouvait même contester sa vérité absolue (p.178). Pour autant, Alexandre Gordon ne sous-estime pas de nouvelles tendances apparues à la limite des années 1950-1960, qui transparaissent dans l'appellation de «Grande » donnée à la Révolution française. Un processus de réhabilitation se déroulait dans les années 1960, à travers l'héroïsation de leaders jacobins. La Révolution française retrouve son statut de révolution "prototype » et Albert Manfred, le leader des historiens de la Révolution de cette époque a eu un grand mérite dans ce domaine. Ainsi, les historiens des années 1960-1970, tout en restant fidèles à la méthodologie marxiste, ont pu avancer des thèses variées et parfois en contradiction les unes avec les autres. Ils se sont notamment engagés dans une polémique sur la nature de la dictature jacobine et du rôle historique des Jacobins. Si A. Manfred et les autres élèves de $\mathrm{N}$. Loukine les considéraient toujours essentiellement, d'après les jugements de Lénine, comme les représentants de la moyenne et petite bourgeoisie, V. Révounenkov les présentaient comme les défenseurs des intérêts de la grande bourgeoisie. Alexandre Gordon étudie minutieusement les péripéties de cette polémique (p. 287-318).

8 Concernant l'attitude des historiens soviétiques envers les approches non marxistes, particulièrement lorsqu'il s'agissait d'analyser l'histoire des masses populaires hors du cadre de la théorie de la lutte des classes, Alexandre Gordon revient sur les réactions de V. Daline envers les derniers ouvrages de R. Cobb. Daline dénonçait «l'évolution » de R. Cobb, et lui reprochait d'avoir laissé de côté l'histoire de la lutte des masses (p. 267). Il montre ainsi très bien qu'on ne considérait comme historiens "progressistes ", en URSS, que ceux qui s'en tenaient à la théorie marxiste. Dans les années suivantes, le pluralisme se développe. On l'aperçoit nettement dans le chapitre que consacre l'auteur à la célébration du Bicentenaire de la Révolution française en URSS ; d'après ses calculs, entre 1985 et 1989 ont paru quatorze monographies et autant de recueils d'articles (p. 327) (voir aussi à ce propos l'information que j'ai publiée dans les AHRF : Chronique 
des ouvrages publiés à l'occasion du bicentenaire de la Révolution en Union Soviétique - 1992, $\mathrm{n}^{\circ} 288$, p. 279-280).

Alexandre Gordon ne doute pas que l'étude de la lutte rurale a été la « contribution la plus importante » de ses prédécesseurs aux études révolutionnaires, citant les noms de N. Loukine et A. Ado (p. 366), dont il a analysé en détail les œuvres au fil de son livre. À proprement parler, cette conclusion me semble limitée, car je suis sûr que la contribution de nos prédécesseurs (V. Daline, A. Ioannissian, G. Tchertkova et d'autres) à l'étude de la pensée communiste et du mouvement des «enragés " (Y. Zakher) ne le cède pas par sa portée à celle citée par l'auteur. Il croit que « les historiens soviétiques et l'historiographie soviétique ont occupé dans son ensemble leur place dans la recherche mondiale de la Révolution française» (p.369). Mais quelle place? Il me semble que les historiens de l'URSS ont occupé une place majeure dans l'historiographie de la Révolution française, surtout si l'on prend en considération leur apport à l'étude de l'époque révolutionnaire vue d'en bas. Il ne faut pas oublier que les représentants les plus célèbres des différentes générations d'historiens français de la Révolution, d'Albert Mathiez à Jacques Godechot, ont constamment regretté de ne pas savoir lire le russe...

Bien d'autres aspects du livre mériteraient d'être présentés ou discutés. Incontestablement, le livre d'Alexandre Gordon est une étude originale, critique et richement documentée, soutenue par la grande érudition de son auteur et son désir sincère de réévaluer de manière impartiale la contribution de ses prédécesseurs à l'histoire de la Révolution française. Il nous invite à repenser la complexité de l'historiographie soviétique, et nous aide à nous orienter dans sa redécouverte. 